\title{
REMOÇÃO DA TURBIDEZ DA ÁGUA USANDO ALOE VERA COMO COAGULANTE NATURAL
}

Rodrigo Babora Borri ${ }^{1}$

Rosane Freire ${ }^{2}$

\section{Welliton Leandro de Oliveira Boina ${ }^{3}$}

\begin{abstract}
RESUMO
O objetivo do trabalho foi avaliar a remoção de turbidez de águas naturais aplicando o extrato glicólico de Aloe vera como agente coagulante. As variáveis investigadas para caracterização das amostras de água bruta e tratada foram turbidez, $\mathrm{pH}$, demanda química de oxigênio e sólidos totais. As condições ótimas de operação foram obtidas por meio de testes em jarro. As condições de operação dos ensaios seguiram as proposições de $\mathrm{Vaz}$ et al. (2010). As dosagens do extrato do coagulante aplicadas

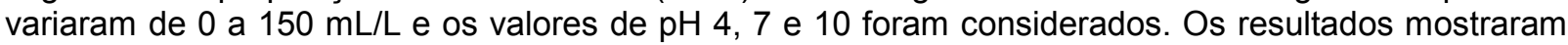
reduções significativas de turbidez em pH 10 e a eficiência média de remoção foi de $75 \%$. A concentração da demanda química de oxigênio e de sólidos totais tiveram os respectivos valores de concentração elevados com o aumento das dosagens aplicadas. Os resultados obtidos permitem sugerir o emprego do Aloe vera como auxiliar de coagulação e estudos visando o detalhamento dos mecanismos de coagulação são incentivados.
\end{abstract}

PALAVRAS-CHAVE: Tratamento de água. Coagulação. Aloe vera.

\section{REMOVAL WATER TURBIDITY USING ALOE VERA AS A NATURAL COAGULANT}

\footnotetext{
${ }^{1}$ Engenheiro Ambiental, Global Solutions Ambiental. Email: rodrigo.babora@hotmail.com

2 Engenheira Ambiental. Mestre e Doutora em Engenharia Química. Professora Assistente Doutora do Departamento de Física, Química e Biologia da Universidade Estadual Paulista, Faculdade de Ciências e Tecnologia, Campus Presidente Prudente-SP. E-mail. rosane@fct.unesp.br

${ }^{3}$ Engenheiro Ambiental. Mestre em Engenharia Urbana. Assistente de Suporte Acadêmico da Universidade Estadual Paulista, Faculdade de Ciências e Tecnologia, Campus Presidente PrudenteSP. E-mail.welliton@fct.unesp.br
} 


\begin{abstract}
The aim of this work was evaluate the removal of turbidity of natural waters applying glycolic extract of Aloe vera as a coagulating agent. The variables investigated for the characterization of samples of natural and treated water were turbidity, $\mathrm{pH}$, chemical oxygen demand and total solids. The optimum operating conditions were obtained by Jar test. Operating conditions of the tests followed the method described in Vaz et al. (2010). The dosage of coagulant extract ranged from 0 to $150 \mathrm{ml} / \mathrm{L}$ and $\mathrm{pH} \mathrm{4,} 7$ and 10 were considered. The results showed significant reductions in turbidity at $\mathrm{pH} 10$ and the average removal efficiency was 75\%. The concentration of chemical oxygen demand and total solids have had their concentration values changed with increasing dosages applied. The results obtained suggest the use of Aloe vera as coagulation aid and studies aimed at detailing the clotting mechanisms are encouraged.
\end{abstract}

KEY-WORDS:Water Treatment. Coagulation. Aloe vera.

\title{
REMOCIÓN DE LA TURBIDEZ DEL ÁGUA USANDO ALOE VERA COMO COALGULANTE NATURAL
}

\section{RESUMEN}

El objetivo de este estudio fue evaluar la remoción de la turbiedad de las aguas naturales aplicando extracto glicólico de Aloe vera como coagulante. Las variables investigadas para la caracterización de las muestras de agua cruda y tratada fueron turbidez, $\mathrm{pH}$, demanda química de oxígeno y sólidos totales. Las condiciones de operación óptimas se obtuvieron por prueba de jarras. Las condiciones de funcionamiento de las pruebas siguieron las proposiciones de Vaz et al. (2010). La dosis de extracto de coagulante varió de 0 a $150 \mathrm{ml} / \mathrm{L}$ y pH 4, se consideraron 7 y 10. Los resultados mostraron reducciones significativas en la turbidez a pH 10 y la eficiencia media de eliminación fue de $75 \%$. La concentración de la demanda química de oxígeno y sólidos totales son los valores respectivos de alta concentración con el aumento de las dosis aplicadas. Los resultados obtenidos sugieren el uso de Aloe vera como ayuda de la coagulación y estudios encaminados a detallar los mecanismos de coagulación se alienta.

PALABRAS-CLAVE Tratamiento del água. Coagulación. Aloe vera

\section{INTRODUÇÃO}

A utilização de coagulantes químicos tem se tornado alvo de reavaliações quanto a sua viabilidade para o tratamento de água e de resíduos líquidos industriais. Richter (2009) expõe que a utilização do sulfato de alumínio no processo de coagulação da água natural permite a ligação química dos produtos da hidrólise com as partículas coloidais do meio, favorecendo, desse modo, a formação de flocos ou mesmo que o íon de alumínio permaneça dissociado na solução. 
A grande preocupação gerada sobre esse aspecto é que a ingestão de determinados elementos químicos oferece um considerável risco à saúde. Pesquisas mostram uma relação entre o elemento alumínio e a incidência de doenças neurológicas (MUISA et al., 2011;WANG et al., 2010; VERÍSSIMO e GOMES, 2008). Além de apresentar risco à saúde, a utilização de coagulantes químicos gera uma elevada produção de lodo nas estações de tratamento de água (ETA) (VARRELI et al., 2009). Esse resíduo, por sua vez, deve ser tratado e descartado de modo seguro e ambientalmente correto, de modo a não causar nenhum dano ao meio.

Assim, nos dias atuais, é necessário que os processos de tratamento de água para abastecimento público se tornem cada vez mais sustentáveis, além de levar em conta as implicações sobre a saúde pública e viabilidade econômica. Tal condição pode ser atingida a partir da utilização de coagulantes naturais, pois são de fácil acesso, não oferecem toxidez ao meio e são de baixo custo, tornando-se uma importante área para a investigação ou pesquisa.

Os principais coagulantes naturais estudados em pesquisas referentes ao tratamento de água são a Moringa Oleifera, os Taninos e a Quitosana (PRITCHARD et al., 2010; HEREDIA et al., 2010; NG et al., 2013). Ao estudar uma proposta com um coagulante diferente, Lima (2007) avaliou a utilização do quiabo (Abelmoschus esculentum, família das Malvaceae) como agente auxiliar de floculação para o tratamento de água. Os resultados encontrados mostraram promissores, apresentado $85 \%$ de remoção de turbidez quando se utilizou $1,25 \mathrm{mg} / \mathrm{L}$ de polímero de quiabo associado a $6 \mathrm{mg} / \mathrm{L}$ de sulfato de alumínio. Esse porcentual é 15\% superior ao resultado obtido utilizando apenas o coagulante químico. De modo semelhante, Verbel et al. (2013) obtiveram uma redução de 93,25\% na turbidez da água do rio Magdalena, Colombia, utilizando como coagulante o gel presente nas folhas do cactus Opuntia.

Com essa perspectiva, o Aloe vera (Aloe barbadensis Miller), família das Liliáceas, pode possuir um potencial significante para coagulação de águas naturais. O Aloe é caracterizado como uma planta perene que possui folhas espessas e 
contém em seu interior uma substância viscosa, o "gel de babosa" (JYOTI NEMA e MITRA 2013).

O gel ou a mucilagem possui em torno de 130 compostos já identificados e que podem ser classificados em vitaminas, aminoácidos, polissacarídeos, enzimas, ácidos graxos, ligninas, saponinas, complexos antraquinônicos, alantoína e sais minerais (JYOTI NEMA e MITRA 2013; PEREIRA E FRASSON, 2007). Essas características conferem uma ampla aplicação na área médica. Contudo, os polímeros presentes são substâncias interessantes para estudos na área de saneamento tendo em vista seu potencial para desestabilizar (coagular) suspensões coloidais presentes nas águas naturais (HESPANHOL, 1982).

Com o exposto, o presente trabalho apresenta uma avaliação preliminar da aplicabilidade do Aloe vera como coagulante de águas naturais. O enfoque principal foi verificar a eficiência de remoção de turbidez em função da dosagem aplicada. Esta pesquisa subsidia o desenvolvimento de estudos que visam, principalmente, à inserção de novas tecnologias e que possuem como base a sustentabilidade do ambiente.

\subsection{Objetivo}

Este trabalho teve como objetivo avaliar a remoção de turbidez de águas naturais aplicando do extrato glicólico de Aloe vera como agente coagulante.

\section{DESENVOLVIMENTO}

Para atingir o objetivo proposto, foram coletadas amostras de água de um manancial de abastecimento público, chamado de Balneário da Amizade, localizado na divisa dos municípios de Álvares Machado-SP e Presidente Prudente-SP (Figura 1). 
Figura 1: Manancial de coleta de água bruta.

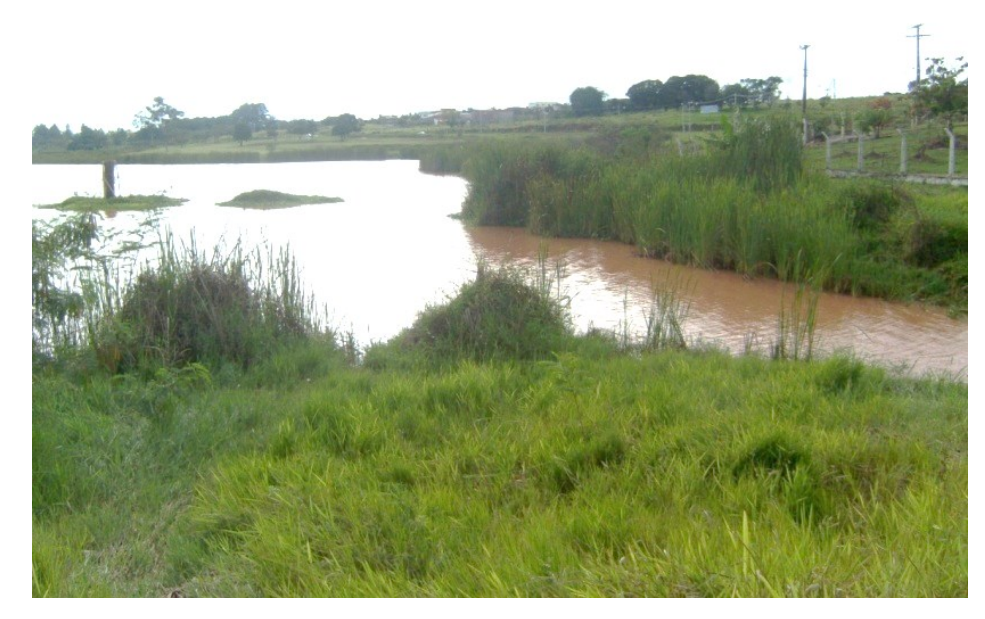

Fonte: Autor, 2013.

As coletas foram realizadas em seis campanhas realizadas durante o período de maio de 2013 a agosto de 2013. O vertedouro da barragem do balneário foi utilizado como ponto amostral. As variáveis investigadas para caracterização das amostras de água bruta foram a turbidez (NTU), potencial hidrogeniônico $(\mathrm{pH})$, demanda química de oxigênio ( $\mathrm{DQO}-\mathrm{mg} / \mathrm{L})$ e sólidos totais ( $\mathrm{mg} / \mathrm{L})$.

Foram utilizados o pHmetro HANNA ${ }^{\circledR}$, modelo $\mathrm{pH} 21 \mathrm{mV}$ meter, e o turbidímetro de bacada microprocessado TB 1000. Os demais procedimentos analíticos seguiram as metodologias e recomendações apresentadas em APHA (1998).

Como não era possível armazenar um volume suficiente de água para que todos os experimentos laboratoriais fossem realizados de forma contínua em cada campanha, realizou-se um sorteio ao acaso de modo que as dosagens fossem aplicadas de forma aleatória às coletas de água realizadas. Todos os ensaios foram executados na Central de Laboratórios de Química, da Faculdade de Ciências e Tecnologia - UNESP, campus de Presidente Prudente-SP.

O agente coagulante utilizado foi o extrato glicólico de Aloe vera comercial. As dosagens aplicadas do extrato variaram de 0 a $150 \mathrm{~mL} / \mathrm{L}$, com intervalos de dosagem 
de $5 \mathrm{~mL}$. A influência do $\mathrm{pH}$ foi analisada a partir dos valores 4,7 e 10, possibilitando analisar o comportamento da substância em estudo em meio levemente ácido, neutro e alcalino. As correções de $\mathrm{pH}$ foram realizadas por meio de uma solução de ácido sulfúrico $0,01 \mathrm{~N}$ e uma solução de hidróxido de sódio a $0,1 \mathrm{~N}$.

A elaboração das superfícies de resposta em relação à aplicação do coagulante natural foi obtida por meio de testes em jarro, utilizando o equipamento Jar-Test Microcontrolado, Milan ${ }^{\circledR}$, modelo JT203 (Figura 2). As condições de operação dos ensaios foram: mistura rápida ocorrendo a $120 \mathrm{rpm}$ durante 1,5 minutos, mistura lenta a $40 \mathrm{rpm}$ durante 15 minutos e, por fim, um tempo de sedimentação de 20 minutos, seguindo as recomendações descritas em Vaz et al. (2010).

Figura 2: Ensaio Jar test.

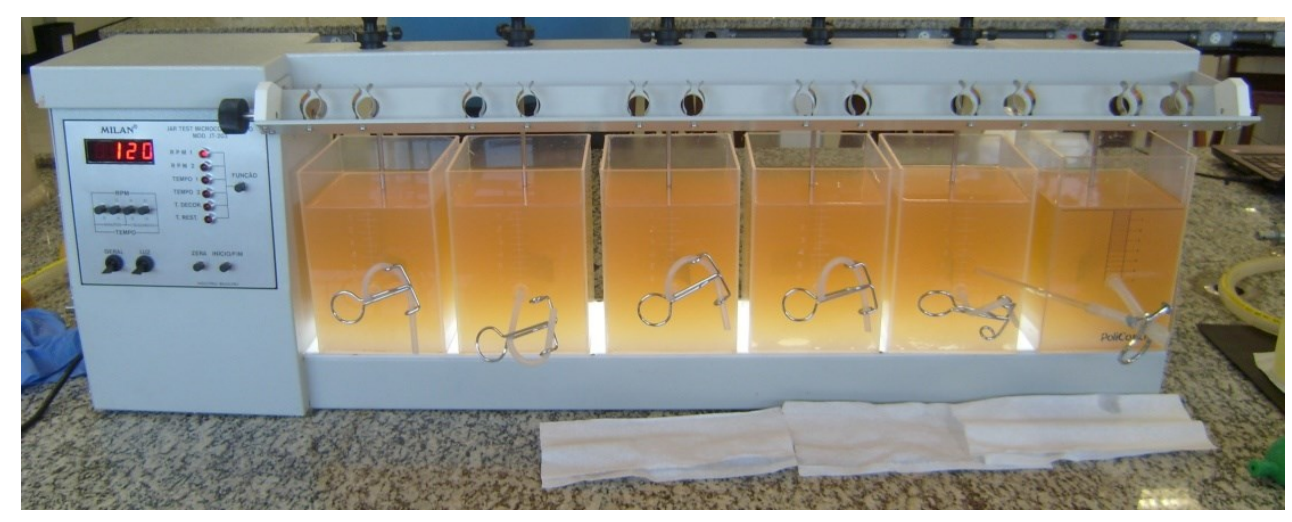

Fonte: Autor, 2013.

A avaliação da eficiência do tratamento proposto foi fornecida por meio da medida de redução de turbidez na água tratada pelo coagulante proposto. A eficiência do processo foi estimada por meio da Equação 1.

$$
E(\%)=\frac{\left(\operatorname{Turb}_{i}-\operatorname{Turb}_{f}\right)}{\operatorname{Turb}_{i}}
$$

em que:

$E(\%)$ corresponde a eficiência de redução de turbidez (\%); 


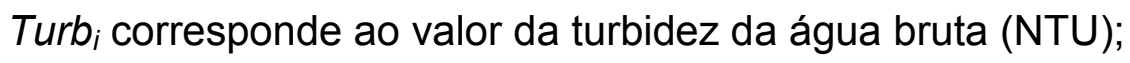

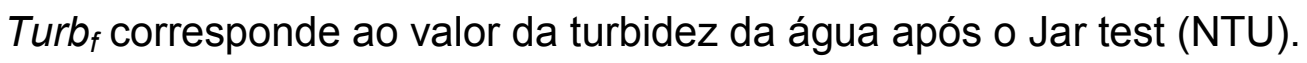

Após o ensaio de jarros, foi selecionada a água tratada no qual se obteve maior redução de turbidez, segundo critérios de $\mathrm{pH}$ e dosagem, para caracterização final quanto ao $\mathrm{pH}$, demanda química de oxigênio ( $\mathrm{DQO}-\mathrm{mg} / \mathrm{L}$ ) e sólidos totais (mg/L). A finalidade principal foi avaliar a interferência do extrato glicólico de Aloe vera na qualidade final da água tratada.

\section{RESULTADOS}

$\mathrm{Na}$ caracterização da água bruta verificou-se que a concentração média de sólidos totais foi de $0,305 \mathrm{mg} / \mathrm{L}$. Quanto ao valor da demanda química de oxigênio, o valor médio da concentração foi de $0,006 \mathrm{mg} / \mathrm{L}$.O valor de $\mathrm{pH}$ da água bruta obtido nas seis coletas apresentou uma variação entre 6,0 e 7,6. Esses resultados conferiram uma característica de neutralidade para as águas do balneário da Amizade durante o período de estudo, indicando pouca interferência da ação antrópica.

A variação da turbidez na água bruta ficou condicionada aos eventos de chuva que antecederam as coletas. De modo geral, dois intervalos principais puderam ser definidos. Em 65,93\% das medições apresentaram turbidez inicial entre 300 e 600 NTU e em $32,97 \%$ das amostras apresentaram valores entre 800 e 1100 NTU. Como exceção, em uma única coleta foi obtido um valor de turbidez igual a 85,6 NTU.

De fato, não há indústrias nas mediações do balneário, contudo, há a presença de condomínios residenciais e muitas áreas em loteamento aberto para a venda. Os resultados encontrados indicam que o manancial recebe uma quantidade significativa de sedimentos, que é intensificada pela ação do escoamento superficial. As áreas loteadas podem ser as principais responsáveis pelos elevados valores encontrados visto que, nesses locais o solo encontrava-se exposto e, assim, com maior suscetibilidade de ser transportado pelas águas da chuva.

Os resultados obtidos no ensaio de coagulação/floculação, apresentados na Tabela 1, mostraram uma forte relação com o pH da água em tratamento. 
Tabela 1: Redução de turbidez com a dosagem de Aloe vera.

\begin{tabular}{|c|c|c|c|c|c|c|c|}
\hline \multirow{2}{*}{$\begin{array}{c}\text { Dosagem } \\
\text { (mL/L) }\end{array}$} & \multicolumn{3}{|c|}{ Redução de Turbidez (\%) } & \multirow{2}{*}{$\begin{array}{c}\text { Dosagem } \\
\text { (mL/L) }\end{array}$} & \multicolumn{3}{|c|}{ Redução de Turbidez (\%) } \\
\hline & pH 4 & $\mathrm{pH} 7$ & $\mathrm{pH} 10$ & & pH 4 & pH 7 & pH 10 \\
\hline 5 & 10,1 & 3,2 & 40,2 & 80 & 19,6 & 22,1 & 73,7 \\
\hline 10 & 14,4 & 14,7 & 77,2 & 85 & 17,9 & 23,6 & 75,2 \\
\hline 15 & 10,2 & 7,4 & 62 & 90 & 18,7 & 21,4 & 74,8 \\
\hline 20 & 16 & 5,8 & 77,5 & 95 & 23 & 23,5 & 74,6 \\
\hline 25 & 10,5 & 6,9 & 78,1 & 100 & 23,2 & 17,4 & 73,8 \\
\hline 30 & 14,8 & 13,3 & 78,5 & 105 & 22,6 & 20,4 & 75,6 \\
\hline 35 & 10 & 12,2 & 60,1 & 110 & 25,2 & 24,8 & 74,9 \\
\hline 40 & 11 & 8,1 & 77,7 & 115 & 23,4 & 22,7 & 75 \\
\hline 45 & 17,9 & 14,2 & 77,7 & 120 & 26,1 & 26,2 & 75,4 \\
\hline 50 & 19,7 & 13,1 & 79,2 & 125 & 26,5 & 27,4 & 75,8 \\
\hline 55 & 24,5 & 20,7 & 78,8 & 130 & 22,2 & 45,8 & 74,3 \\
\hline 60 & 24,9 & 20 & 78,3 & 135 & 21,7 & 47,2 & 76,3 \\
\hline 65 & 18,5 & 24,5 & 73,8 & 140 & 25,1 & 78,6 & 76,7 \\
\hline 70 & 15,9 & 15,9 & 74 & 145 & 27,2 & 60,9 & 75,4 \\
\hline 75 & 19,1 & 24,5 & 74,2 & 150 & 25 & 60,8 & 75,7 \\
\hline
\end{tabular}

Fonte: Autor, 2013.

Em pH 4, o tratamento proposto teve baixa eficiência na redução de turbidez, independente da dose do extrato glicólico aplicado. A média de remoção foi de 19,8\% com variação de $\pm 5 \%$. Em meios ácidos, pode ocorrer a neutralização da carga elétrica das partículas coloidais pelas espécies positivas dos polímeros do Aloe vera. Neste caso, como prevê Di Bernardo e Dantas (2005), a variação da dosagem do coagulante não resultará na redução da turbidez. Essa afirmação explicaria os resultados encontrados em $\mathrm{pH} 4$, no entanto, maiores investigações devem ser feitas. A confirmação desse efeito deve ser constatada pelo potencial zeta do meio, contudo, por motivos técnicos, essas medidas não puderam ser realizadas.

$\mathrm{Em} \mathrm{pH} \mathrm{7,} \mathrm{o} \mathrm{tratamento} \mathrm{teve} \mathrm{uma} \mathrm{redução} \mathrm{média} \mathrm{do} \mathrm{valor} \mathrm{de} \mathrm{turbidez} \mathrm{de} 25 \%$. Contudo, em dosagens elevadas verificaram-se reduções superiores a $60 \%$. Uma eficiência máxima de $78,6 \%$ de remoção foi atingida na dosagem de $140 \mathrm{~mL} / \mathrm{L}$. Neste 
caso, os resultados obtidos poderiam ser explicados a partir de uma relação estequiométrica entre a dosagem do Aloe e a concentração das partículas do meio. Logo, faz igualmente necessário aprofundar no detalhamento existente entre relações identificadas, pois, considerando resultados apresentados na Tabela 1, pode existir um grande intervalo de dosagem no qual a reestabilização do sistema coloidal é dominante.

$\mathrm{Em} \mathrm{pH} 10$ foram verificados as maiores reduções de turbidez, apresentando média de 75,0 4 \% . Esses resultados não mostraram dependência com a dosagem do estrato glicólico de Aloe aplicado. Contudo, não foi verificada a formação de precipitados de forma visualmente nítida e sim, a presença de pequenos aglomerados que permaneceram suspensos no meio.

Em teoria, o meio alcalino favorece a formação de hidróxidos pela hidrólise do agente coagulante. Os compostos hidrolisados, ao interagir com as partículas do meio, formam precipitados, caracterizando o mecanismo de varredura (Di BERNARDO E DANTAS, 2005). Como o coagulante utilizado possui cadeias poliméricas, o mecanismo de adsorção de partículas no meio pode ser sido favorecido, mas não suficiente para formar pontes entre a superfície a qual está aderido e outras partículas. Os valores de turbidez encontrados nesse ensaio podem ser explicados pela ação do fenômeno supracitado. Logo, o emprego do Aloe vera como agente auxiliar de coagulação/floculação deve ser investigado.

Por fim, as amostras dos ensaios com as dosagens 20,25 e $30 \mathrm{ml} / \mathrm{L}$ de extrato glicólico em pH 10 foram levadas para caracterização final do tratamento. Em relação às análises de sólidos totais e demanda química de oxigênio houve a elevação concentração conforme se eleva o valor do coagulante aplicado. Como a sedimentação não foi evidente, a massa do extrato glicólico adicionado passou a ser constituinte da massa total da amostra, justificando os resultados encontrados.

\section{CONCLUSÃO}


A utilização do extrato de glicólico de Aloe vera para o tratamento de água mostrou-se promissor, uma vez que foram obtidas reduções do parâmetro turbidez em todos os ensaios experimentais realizados. $O$ ensaio em meio alcalino foi aquele em que foi obtida a maior eficiência de redução de turbidez das águas naturais estudadas. Os resultados obtidos permitem sugerir o emprego do Aloe vera como auxiliar de coagulação e estudos visando o detalhamento dos mecanismos de coagulação são incentivados.

\section{RECOMENDAÇÕES}

O presente trabalho apresentou um estudo preliminar sobre a eficiência de redução da turbidez de águas naturais utilizando o Aloe vera como agente coagulante. Com os resultados obtidos recomenda-se um estudo aprofundado sobre a interação do potencial hidrogeniônico - dosagem aplicada - partículas, realizando medições do potencial elétrico da solução. Espera-se assim, elucidar os mecanismos de coagulação de modo que tenha maior clareza dos processos químicos e físicos envolvidos.

\section{REFERÊNCIAS}

APHA. American Public Health Association. Standard methods for the examination of water and wastewater. American Public Health Association, 1998, 1600 p.

Di BERNARDO, L., DANTAS, A.D.B. Métodos e técnicas de tratamento de água. Vol $1,2^{\mathrm{a}}$ Ed. São Carlos: RIMa, 2005. 784p.

HEREDIA, J.B., SÁNCHES-MARTÍN, J., GÓMEZ-MUÑOZ, M.C. New coagulant agents from tannin extracts: preliminary optimisation studies. Chemical Engineering Journal, v. 162, n. 3, p. 1019-1025, 2010.

HESPANHOL, I. Cinética da floculação de suspensões coloidais com polieletrólitos naturais. São Paulo: Universidade de São Paulo, 1982, 248p.

JYOTI NEMA, S.K.S., MITRA, N.G. Chemical composition of Aloe ferox under stress of soil pH and desiccation. International Jounal of Chemistry, v.3, 2013.

LIMA, G.J.A. Uso de polímero natural do quiabo como auxiliar de floculação e filtração em tratamento de água e esgoto. 2007. 154f. Dissertação (Mestrado em Engenharia Ambiental, Controle da Poluição Urbana e Industrial) - Universidade do Estado do Rio de Janeiro, Rio de Janeiro. 
MUISA, N., HOKOA, Z., CHIFAMBA, P. Impacts of alum residues from Morton Jaffray Water Works on water quality and fish, Harare, Zimbabwe. Physics and Chemistry of the Earth, Parts A/B/C, v.36, n.14-15, p. 853-864, 2011.

NG, M., LIU, S., CHOW, C.W., DRIKAS, M.; AMAL, R.; LIM, M. Understanding effects of water characteristics on natural organic matter treatability by $\mathrm{PACl}$ and a novel PACl chitosan coagulants. Journal of Hazard Materials, v.263, pt 2, p. 718-725, 2013.

PEREIRA, D.C., FRASSON, A.P.Z. Uso doa Aloe vera em produtos farmacêuticos e análise da estabilidade físico-química de creme aniônico contendo extrato glicólico desta planta. Revista Contexto \& Saúde, UNIJUÍ, v.6, n.12, p. 27-34, 2007.

PRITCHARD, M., CRAVEN, T., MKANDAWIRE, T., EDMONDSON, A.S., O'NEILL, J.G.A Comparision between Moringa oleifera and chemical coagulants in the purification of drinking water - na alternative sustainable solution for developing countries. Physics and Chemistry of the Earth, Parts A/B/C, v.35, n.13-14, p. 798-805, 2010.

RICHTER, C.A. Água: métodos e tecnologia de tratamento. São Paulo: Edgar Blücher, 2009, 340p.

VARRELI, D.I., DIXON, D.R., SCALES, P.J. Effect of coagulation conditions on the dewatering properties of sludges produced in drinkg water treatment. Colloids and Surfaces A: Physicochemical and Engineering Aspects, v.348, n.1-3, p. 14-23, 2009.

VAZ, L.G.L., KLEN, M.R.F., VEIT, M.T., SILVA, E.A., BARBIERO, T.A., BERGAMASCO, R. Avaliação da eficiência de diferentes agentes coagulantes na remoção de cor e turbidez em efluente de Galvanoplastia. Eclética Química, v.35, n.4, p. 45-54, 2010.

VERBEL, R.E.O., MARTíNEZ, I.D.M., GAZABÓN, L.E.M. Remoción de la turbidez del água del rio Magdalena usando el mucílado del nopal Opuntia fícus-indica. Produción + Limpia, v. 8, n.1, p. 19-27, 2013.

VERÍSSIMO, M.I., GOMES, M.T. The quality of our drinking water: aluminium determination with na acoustic wave sensor. Analytica Chimica Acta, v.617, n. 1-2, p. 162-166, 2008.

WANG, W., LI, H., LIU, Y. Spatial variations of aluminum species in driking water supplies in Xi'an studied applying geographic information system. Journal of Environmental Science (China), v.22, n. 4 , p. $519-525,2010$ 